\title{
Effects of arachidonic acid on the concentration of hydroxyeicosatetraenoic acids in culture media of mesenchymal stromal cells differentiating into adipocytes or osteoblasts
}

\author{
Antonio Casado-Díaz • Carlos Ferreiro-Vera • \\ Feliciano Priego-Capote • Gabriel Dorado • \\ María Dolores Luque-de-Castro · José Manuel Quesada-Gómez
}

Received: 28 June 2013/Accepted: 6 November 2013/Published online: 14 December 2013

(C) Springer-Verlag Berlin Heidelberg 2013

\begin{abstract}
Metabolites derived from the polyunsaturated fatty acids (PUFA) may modulate the mesenchymal stromal cell (MSC) differentiation. Such cells can differentiate into different cellular types, including adipocytes and osteoblasts. Aging favors the bone marrow MSC differentiation toward the former, causing a loss of bone density associated with pathologies like osteoporosis. The omega-6 arachidonic acid (AA) favors MSC adipogenesis to a greater extent than omega-3 eicosapentaenoic acid (EPA) and docosahexaenoic acid (DHA). In this work, we study the joint action of both PUFA. Thus, not induced and induced to adipocyte or osteoblast MSC were treated with $20 \mu \mathrm{M}$ of each PUFA (either AA, AA + DHA or $\mathrm{AA}+\mathrm{EPA})$. The expression of osteogenic and
\end{abstract}

Antonio Casado-Díaz and Carlos Ferreiro-Vera have contributed equally to the experimental work.

A. Casado-Díaz · C. Ferreiro-Vera · F. Priego-Capote ·

M. D. Luque-de-Castro · J. M. Quesada-Gómez ( $\square)$

Instituto Maimonides de Investigación Biomedica (IMIBIC).

Hospital Universitario Reina Sofía de Córdoba,

Universidad de Córdoba, 14004 Córdoba, Spain

e-mail:md1qugoj@uco.es; jmquesada@uco.es

C. Ferreiro-Vera $\cdot$ F. Priego-Capote $\cdot$ M. D. Luque-de-Castro Departmento de Química Analítica. Anexo C-3. Campus de Rabanales, Universidad de Córdoba, 14071 Córdoba, Spain

G. Dorado

Departamento de Bioquímica y Biología Molecular, Campus Rabanales C6-1-E17, Campus de Excelencia Internacional Agroalimentario (ceiA3), Universidad de Córdoba,

14071 Córdoba, Spain

J. M. Quesada-Gómez

Unidad de Metabolismo Mineral. Servicio de Endocrinología y

Nutrición. Hospital Universitario Reina Sofía de Córdoba,

Universidad de Córdoba, 14004 Córdoba, Spain adipogenic molecular markers, the alox $15 b$ lipoxygenase gene expression and the 5-, 8-, 11-, 12- and 15-hydroxyeicosatetraenoic acids (HETE) derived from the AA metabolism in the culture media were determined. The results show that the adipogenesis induction of AA is not suppressed by the joint presence of EPA and DHA. In fact, both increased the adipogenic effect of AA on MSC differentiated into osteoblasts. The different HETE concentrations increased in cultures supplemented with AA, albeit such concentrations were lower in the cultures induced to differentiate, mainly at day 21 after the induction. Furthermore, the reduction in the HETE concentration was correlated with a higher expression of the alox $15 \mathrm{~b}$ gene. These results highlight the PUFA metabolism differences between uninduced and induced MSC to differentiate into adipocytes and osteoblasts, besides the relevant role of the lipoxygenase gene expression in adipogenesis induction.

Keywords Omega-6 - Omega-3 - Lipoxygenase ·

Osteogenesis · Adipogenesis .

Hydroxyeicosatetraenoic acid

\section{Introduction}

Omega-6 (n-6) and omega-3 (n-3) polyunsaturated fatty acids (PUFA) are termed "essential" because they are required nutrients, yet the human metabolism cannot synthesize them, requiring their ingestion in the diet (Salari et al. 2008). The main essential fatty acids are linoleic acid (18:2n-6; LA) and $\alpha$-linolenic acid (18:3n-3; ALA). They are absorbed and metabolized into longer-chain PUFA containing 20 or more carbon atoms of the $n-6$ group, such as arachidonic acid (20:4n-6; AA), or the $n-3$ group, such 
as eicosapentaenoic acid (20:5n-3; EPA) and docosahexaenoic acid (22:6n-3; DHA).

The AA is part of the membrane phospholipids and may be reacylated or oxidatively metabolized through the cyclooxygenase (COX) or lipoxygenase (LOX) pathways. The enzyme phospholipase A2 (PLA2) initiates the COX or LOX pathway by catalyzing the hydrolysis of AA from the membrane phospholipids. LOX acts on liberated free AA to generate hydroperoxyeicosatetraenoic acids, which are unstable intermediates, being further either hydrolyzed into hydroxyeicosatetraenoic acids (HETE) via lipoxygenase catalysis or by non-enzymatic oxidative reactions (Niki et al. 2005), or enzymatically converted into leukotrienes such as 5(6)-epoxy(oxido)eicosatetraenoic acid (leukotriene A4; LTA4) and lipoxins, which may be involved in important physiological events such as inflammation (Wahli and Michalik 2012). On the other hand, the HETE are PPAR $\gamma$ agonists and may influence cellular differentiation (Ban et al. 2011; Yu et al. 1995). Additionally, LTA4 may be further converted into either dihydroxyeicosatetraenoic acid (LTB4) by an epoxide hydrolase or hydroxy-glutathionyleicosatetraenoic acid (LTC4) by a glutathione transferase (Needleman et al. 1986).

The metabolism of fatty acids by the COX pathway leads to the formation of the prostanoids, including prostaglandins (PG) and thromboxanes (TX). Although great attention has been focused on the COX-derived PG so far, emerging evidence suggests that LT as well as HETE LOX-catalyzed products may also exert profound biological effects on inflammation processes, being involved in the development and progression of specific human diseases, such as colorectal or pancreatic cancers (Comba and Pasqualini 2009; Di Mari et al. 2007). In addition to their inflammatory role, 12- and 15-lipoxygenases have been reported as promoters of insulin resistance associated with obesity (Chakrabarti et al. 2009) and osteoporosis (Almeida et al. 2009). Interestingly, the same enzymes participating in the metabolism of omega- 6 are involved in the metabolism of omega-3. Thus, there is a competition between such substrates and, consequently, the production of alternative metabolites, depending on the concentration of each PUFA: prostaglandins series 2, thromboxanes series 2, leukotrienes series 4 and lipoxins for omega- 6 fatty acids; or prostaglandins series 3 , thromboxanes series 3 , leukotrienes series 5 and resolvins for omega-3 fatty acids (Schmitz and Ecker 2008).

Several PUFA have shown to affect bone cells via different cellular signaling pathways or growth factors, thereby modulating bone formation, reabsorption and density in different animals, including humans (Jarvinen et al. 2012; Moon et al. 2012; Rahman et al. 2009; Salari et al. 2008). On the other hand, fatty acids play a central role in the induction of mesenchymal stromal cells (MSC) to adipocyte differentiation, as well as in adipose tissue hypertrophy, because they have the ability to activate the peroxisome proliferator-activated receptor gamma $(\operatorname{PPAR} \gamma)$, which is a transcription factor inducing adipogenesis (Lopez et al. 2003; Shillabeer and Lau 1994). We have previously shown that AA promotes a greater degree of adipocyte differentiation of MSC than DHA or EPA (Casado-Diaz et al. 2013). Thus, not only the quantity of ingested fats, but also the nature of their fatty acid residues is of pivotal importance for human health. In fact, the nutritional and metabolic factors, which modulate the PPAR $\gamma$ and therefore the MSC differentiation, such as the amount and nature of PUFA, may influence the bone health (Chen et al. 2013). Besides, aging favors the differentiation of the bone marrow MSC into adipocytes instead of osteoblasts (Moerman et al. 2004). This contributes to the bone mass loss in elderly people, since their ability to generate new bone is reduced.

Therefore, since the hydroxyeicosatetraenoic acids generated from the metabolism of polyunsaturated fatty acids are involved in different biological processes, including the modulation of PPAR $\gamma$, the goal of this research was to study the effects of polyunsaturated fatty acids on the production of hydroxyeicosatetraenoic acids in the culture media of uninduced and induced MSC into osteoblasts or adipocyte differentiation, thus contributing to a better understanding of the effect of PUFA on the metabolism and differentiation of MSC, including their putative role in the bone formation, which has a significant potential interest for the prevention of and treatment for bone diseases, such as osteoporosis.

\section{Materials and methods}

\section{Materials}

Culture flasks and plates were purchased from Nunc (Kamstrupvej, Denmark). The alpha-minimal essential medium ( $\alpha$-MEM), fetal bovine serum (FBS), trypsin and ultraglutamine were from Lonza (Basel, Switzerland). Antibiotics, fibroblast growth factor (bFGF), osteogenic and adipogenic inductors, polyunsaturated fatty acids (AA, DHA and EPA) and reagents for oil red histochemical staining were purchased from Sigma-Aldrich (Saint Louis, MO, USA). The easy-spin Total RNA Extraction Kit, iScript cDNA Synthesis Kit and SYBR Green I Master were from iNtRON Biotechnology (Gyeonggi-do, Korea), Bio-Rad (Hercules, CA, USA) and Roche (Mannheim, Germany), respectively. The HETE standards corresponding to 5-hydroxy-6E,8Z,11Z,14Z-eicosatetraenoic acid (5-HETE), 8-hydroxy-5Z,9E,11Z,14Z-eicosatetraenoic acid (8-HETE), 11-hydroxy-5Z,8Z,12E,14Z-eicosatetraenoic 
acid (11-HETE), 12-hydroxy-5Z,8Z,10E,14Z-eicosatetraenoic acid (12-HETE) and 15-hydroxy-5Z,8Z,11Z,13E-eicosatetraenoic acid (15-HETE) were purchased from Cayman Chemicals (Ann Arbor, MI, USA). The cartridge HySphere C18-EC $(8 \mu \mathrm{m}$ and $10 \times 2.0 \mathrm{~mm})$ and chromatographic column $(3 \mu \mathrm{m}$ and $150 \times 4.6 \mathrm{~mm})$ were from Spark Holland (Emmen, The Netherlands) and Phenomenex (Torrance, CA, USA), respectively.

RNA was quantified at $260 \mathrm{~nm}$ with a NanoDrop ND1000 spectrophotometer (Thermo Fisher Scientific, Lenexa, KS, USA), and the quantitative real-time PCR (QRT-PCR) was performed on a LightCycler 480 real-time PCR thermocycler (Roche). The cultures stained with oil red were visualized using an Eclipse TI inverted microscope from Nikon (Melville, NY, USA), and the staining was quantified after elution with a PowerWave XS microplate reader from BioTek (Winooski, VT, USA). The HETE determinations were carried out with a Midas autosampler and Prospekt-2 System from Spark Holland (Emmen, The Netherlands) for solid-phase extraction (SPE), online connected to a 1,200 series liquid chromatography (LC) system from Agilent (Palo Alto, CA, USA), by which the chromatographic eluate was directly transferred into a 6,410 triple quadrupole detector (QqQ), furnished with an electrospray ion (ESI) source from the same manufacturer.

\section{Methods}

\section{Bone marrow cell cultures}

The mesenchymal stromal cells were obtained from bone marrows of three healthy donors (two females aged 18 and 23 and a male aged 31 years) after their written consent at the Bone Marrow Transplantation Program of the Hematology Service of the Reina Sofía University Hospital in Cordoba (Spain). The MSC were isolated and cryopreserved as we have previously reported (Casado-Diaz et al. 2013). The experiments were performed with a pool of cells obtained from such three donors.

The MSC were seeded in $75-\mathrm{cm}^{2}$ flasks containing a-MEM culture medium with $10 \%$ of FBS and $2 \mathrm{mM}$ of ultraglutamine, as well as $100 \mathrm{U}$ of ampicillin, $0.1 \mathrm{mg}$ of streptomycin $/ \mathrm{ml}$ and $1 \mathrm{ng} / \mathrm{ml}$ of bFGF. The cells were grown at $37{ }^{\circ} \mathrm{C}$ in $5 \% \mathrm{CO}_{2}$, replacing the cell culture medium every 3 days. The experiments were carried out trypsinizing near-confluent cultures and reseeding the cells at about 500 cells $/ \mathrm{cm}^{2}$ in the same culture medium described above, using P6 or P12 multiwell culture plates.

\section{Osteogenic and adipogenic differentiation}

The cells were seeded on 6- or 12-well plates and grown in culture medium until 80-90\% confluency. Then, the bFGF was withdrawn and the medium was supplemented with either $10 \mathrm{nM}$ of dexamethasone, $0.2 \mathrm{mM}$ of ascorbic acid and $10 \mathrm{mM}$ of $\beta$-glycerophosphate (to induce the cells to differentiate into osteoblasts), or $0.5 \mu \mathrm{M}$ of dexamethasone, $0.5 \mathrm{mM}$ of isobutylmethylxanthine and $50 \mu \mathrm{M}$ of indomethacin (to induce the adipogenesis differentiation). The control MSC (not induced to differentiate) were maintained in basal medium ( $\alpha$-MEM plus $10 \%$ of FBS, but without bFGF).

\section{Polyunsaturated fatty acid treatments}

Human MSC were induced to differentiate into either osteoblasts or adipocytes in the presence or absence (controls) of $20 \mu \mathrm{M}$ of AA. The AA was added either alone or in the presence of EPA or DHA at the same concentration. Furthermore, MSC not induced to differentiate (controls) were treated with the same concentrations of PUFA listed above, to evaluate the influence of the cellular type on the analyzed metabolites. The cultures were grown for 21 days, taking samples of both the cell culture medium (AA metabolism) and the cells (RNA isolation and gene expression) at different times. They were stored at $-80{ }^{\circ} \mathrm{C}$ until needed for the analyses.

\section{$R N A$ extraction, reverse transcription and QRT-PCR}

Total RNA from cell cultures was isolated and quantified as previously described and further segregated using agarose gel electrophoresis to check its integrity. The RNA transcriptions were performed in a volume of $20 \mu \mathrm{l}$, using $1 \mu \mathrm{g}$ of total RNA and the iScript cDNA Synthesis Kit. The QRT-PCR was performed with the SYBR Green I Master, using $1 \mu \mathrm{l}$ of cDNA and 10 pmol of each primer corresponding to the tested genes (Table 1), as previously indicated. The relative quantity of the mRNA for each sample was calculated using the copy threshold $(\mathrm{Ct})$ value. The polymerase RNA II polypeptide A mRNA (polr $2 a$ ) was used as housekeeping gene for the normalization. The stability of the housekeeping gene was tested with the BestKeeper application (Pfaffl et al. 2004). The amplification profile included one cycle at $95{ }^{\circ} \mathrm{C}$ for $10 \mathrm{~min}$ and 40 three-step cycles: $94{ }^{\circ} \mathrm{C}$ for $10 \mathrm{~s}, 65^{\circ} \mathrm{C}$ for $15 \mathrm{~s}$ and $72{ }^{\circ} \mathrm{C}$ for $15 \mathrm{~s}$. The results were analyzed with the LightCycler software 1.5.0, using the second-derived method for the $\mathrm{Ct}$ calculation.

\section{Histochemical staining}

Fat accumulation in MSC cultures was tested using oil red staining. Cells were fixed with $3.7 \%$ formaldehyde for $20 \mathrm{~min}$, rinsed with isopropanol $60 \%$ in water and subsequently stained for 15-20 min with a mixture of $60 \%$ of 
Table 1 PCR primer sequences

\begin{tabular}{|c|c|c|c|}
\hline Gene category & Gene name & Primer sequence $\left(5^{\prime} \rightarrow 3^{\prime}\right)$ & $\begin{array}{l}\text { Product } \\
\text { size }(b p)\end{array}$ \\
\hline \multirow[t]{6}{*}{ Adipogenic } & \multirow[t]{2}{*}{ Peroxisome proliferator-activated receptor gamma $2($ ppar $-\gamma 2)$} & GCGATTCCTTCACTGATACACTG & \multirow[t]{2}{*}{136} \\
\hline & & GAGTGGGAGTGGTCTTCCATTAC & \\
\hline & \multirow[t]{2}{*}{ Lipoprotein lipase $(l p l)$} & AAGAAGCAGCAAAATGTACCTGAAG & \multirow[t]{2}{*}{113} \\
\hline & & CCTGATTGGTATGGGTTTCACTC & \\
\hline & \multirow[t]{2}{*}{ Glycerol-3-phosphate dehydrogenase 1 (gpdl) } & ATACAGCATCCTCCAGCACAAG & \multirow[t]{2}{*}{120} \\
\hline & & GGATGATTCTGCAGGCAGTG & \\
\hline \multirow[t]{6}{*}{ Osteogenic } & \multirow[t]{2}{*}{ Runt-related transcription factor 2 (runx 2$)$} & TGGTTAATCTCCGCAGGTCAC & \multirow[t]{2}{*}{143} \\
\hline & & ACTGTGCTGAAGAGGCTGTTTG & \\
\hline & \multirow[t]{2}{*}{ Alkaline phosphatase (alp) } & CCAACGTGGCTAAGAATGTCATC & \multirow[t]{2}{*}{175} \\
\hline & & TGGGCATTGGTGTTGTACGTC & \\
\hline & \multirow[t]{2}{*}{ Osteocalcin $(o c)$} & CCATGAGAGCCCTCACACTCC & \multirow[t]{2}{*}{258} \\
\hline & & GGTCAGCCAACTCGTCACAGTC & \\
\hline \multirow[t]{8}{*}{ Alox } & \multirow[t]{2}{*}{ Arachidonate 5-lipoxygenase (alox5) } & AAGGGCGTGGTGACCATTGAG & \multirow[t]{2}{*}{190} \\
\hline & & CGAGGTTCTTGCGGAATCGG & \\
\hline & \multirow[t]{2}{*}{ Arachidonate 12-lipoxygenase (alox12) } & TCTCTATGCCCATGATGCTTTACG & \multirow[t]{2}{*}{217} \\
\hline & & GGTGAGGAAATGGCAGAGTTGAC & \\
\hline & \multirow[t]{2}{*}{ Arachidonate 15-lipoxygenase (alox15) } & ACACTTGATGGCTGAGGTCATTG & \multirow[t]{2}{*}{155} \\
\hline & & GGTCGAAAATTCCCATGTCAGAG & \\
\hline & \multirow[t]{2}{*}{ Arachidonate 15-lipoxygenase, type B (alox15b) } & CAAGGGGCTCTGGAGGAGTC & \multirow[t]{2}{*}{120} \\
\hline & & AGGAACTGGGAGGCGAAGAAG & \\
\hline \multirow[t]{2}{*}{ Housekeeping } & \multirow[t]{2}{*}{ Polymerase RNA II polypeptide A (polr2a) } & TTTTGGTGACGACTTGAACTGC & \multirow[t]{2}{*}{125} \\
\hline & & CCATCTTGTCCACCACСТCTTC & \\
\hline
\end{tabular}

$0.3 \%$ (w/v in isopropanol) of oil red agent plus $40 \%$ of distilled water. Cells were then rinsed with water and airdried. Next, the oil red stain was extracted with isopropanol at room temperature for $10 \mathrm{~min}$, and the absorbance of the resulting solution was measured at $510 \mathrm{~nm}$ to quantify the fat formation. The oil red measurements were normalized with the number of cells: The cell cultures were stained with $0.1 \%$ crystal violet in $10 \%$ ethanol for $20 \mathrm{~min}$ and washed with water, and then the crystal violet was extracted with $10 \%$ acetic acid for $20 \mathrm{~min}$. The absorbance of the resulting solution was measured at $590 \mathrm{~nm}$, and the amount of fat was expressed as $A_{510 n m} / A_{590 n m}$. The cultures were visualized using an inverted microscope.

\section{HETE concentrations in culture media}

We had previously developed the analytical methodology used for the determination of the target compounds (Ferreiro-Vera et al. 2011). Briefly, $100 \mu \mathrm{l}$ of the culture media was injected into a Midas autosampler and Prospekt2 system for solid-phase extraction (SPE). The system was online connected to a 1,200 series liquid chromatography (LC) system with the chromatographic eluate being directly introduced into a 6,410 triple quadrupole detector (QqQ), furnished with an electrospray ion (ESI) source. Then, the SPE-LC-MS/MS analysis was started with the solvation of stationary phase of cartridge HySphere C18-EC $(8 \mu \mathrm{m}$ and $10 \times 2.0 \mathrm{~mm})$ with $2 \mathrm{ml}$ of methanol. Conditioning and equilibration were accomplished using $2 \mathrm{ml}$ of water, with subsequent sample loading into the cartridge with $2 \mathrm{ml}$ of water. The target compounds were retained in the sorbent, and $2 \mathrm{ml}$ of a $20 \%$ methanol aqueous solution was used as rinsing solution to remove the interferents. The chromatography started by switching the left-clamp valve and eluting the content of the cartridge for $30 \mathrm{~s}$ into the chromatographic column $(3 \mu \mathrm{m}$ and $150 \times 4.6 \mathrm{~mm})$, with the isocratic mobile phase made of 76:22:2:0.02 (v/v) methanol/water/acetonitrile/acetic acid, which was injected by the chromatographic pump at a flow of $0.8 \mathrm{ml} / \mathrm{min}$. The column compartment was maintained at $25{ }^{\circ} \mathrm{C}$.

Mass spectrometry detection was performed in negative electrospray ionization mode at $4 \mathrm{kV}$ capillary voltage, $315^{\circ} \mathrm{C}$ source temperature and 60 psi pressure nebulizer. Nitrogen was used as desolvation gas with a flow of 10 $\mathrm{ml} / \mathrm{min}$. The selected voltage for efficient filtration of the 
Table 2 Optimization of MS/MS step for qualitative and quantitative determination of the HETE

\begin{tabular}{llllll}
\hline Analyte & Precursor ion $(\mathrm{m} / \mathrm{z})$ & Voltage MS1 $(\mathrm{eV})$ & Product ion $(\mathrm{m} / \mathrm{z})$ & Collision energy $(\mathrm{eV})$ & Quantitation transition $(\mathrm{m} / \mathrm{z})$ \\
\hline 15-HETE & 319.2 & 80 & 175.2 & 10 & $319.2 \rightarrow 175.2$ \\
12-HETE & 319.2 & 80 & 179.2 & 10 & $319.2 \rightarrow 179.2$ \\
11-HETE & 319.2 & 80 & 167.2 & 15 & $319.2 \rightarrow 167.2$ \\
8-HETE & 319.2 & 80 & 155.2 & 10 & $319.2 \rightarrow 155.2$ \\
5-HETE & 319.2 & 80 & 115.2 & 15 & $319.2 \rightarrow 115.2$ \\
\hline
\end{tabular}

precursor ions in the first quadrupole collision energy fragmented the precursor ions by collision-induced dissociation (CID). The product ions selected as quantification and qualifier ions in the selected reaction monitoring (SRM) method for each analyte are shown in Table 2. The dwell time was fixed at $200 \mathrm{~ms}$ for each SRM transition. The entire analytical process was completed within $30 \mathrm{~min}$.

Statistical analyses

Three independent biological experiments were conducted and three analytical replicates were measured, obtaining the corresponding means. All $p$ values were calculated using the analysis of variance (ANOVA) and Fisher's projected least significant difference (PLSD) tests. A value of $p<0.05$ was considered statistically significant.

\section{Results}

The AA effect on the adipogenesis is not modified by the presence of EPA or DHA

The presence of $20 \mu \mathrm{M}$ of AA increased the adipogenesis on the MSC induced to differentiate into adipocytes, as shown by the increase in the gene expression of the ppar 2 , lipoprotein lipase $(l p l)$ and soluble glycerol-3-phosphate dehydrogenase 1 ( $g p d l$ ) adipogenic genes, in relation to the control cultures at 7 and 21 days after the adipocyte differentiation induction (Fig. 1a). Besides, the number of adipocytes increased in such cultures, as well as their fat vesicles, which was quantified by oil red staining (Fig. 1b, c).

The cultures induced to differentiate into adipocytes in the presence of AA were also supplemented with $20 \mu \mathrm{M}$ of omega-3 DHA or EPA, to ascertain their putative influence on such AA adipogenesis induction. The presence of the omega-3 fatty acids only reduced the expression of the gpdl gene at day 21 of the treatment, in relation to the cultures supplemented with AA only (Fig. 1a), without significant changes in the expression of the other studied genes. On the other hand, the presence of omega- 3 fatty acids did not significantly alter the number of adipocytes and fat vesicles in the cultures supplemented with AA (Fig. 1b, c). Therefore, the DHA or EPA concentrations used did not modify the effect of AA on the MSC differentiating into adipocytes.

Effect of the AA on the MSC differentiation into osteoblasts in the absence and presence of omega-3 fatty acids

The presence of AA repressed the runt-related transcription factor 2 (runx2) and alkaline phosphatase (alp) osteoblastogenesis early-expression genes in the MSC at day 7 after the treatment and osteoblast induction (Fig. 2a). Yet, the AA did not have a significant effect on the induction of the osteocalcin (oc) osteoblastogenesis late-expression gene (Fig. 2a). The joint supplementation of AA plus DHA, or AA plus EPA, significantly increased the gene expression of alp gene at day 21 on the MSC induced to differentiate into osteoblasts, in relation to the cultures supplemented with AA only, without affecting the gene expression of runx 2 or $o c$.

The AA produced the appearance of cells with adipocyte phenotype in the MSC cultures induced to differentiate into osteoblasts, including the accumulation of fat vesicles (Fig. 2b). The joint supplementation of AA plus DHA (or AA plus EPA) significantly increased the number of adipocytes, in relation to the addition of AA only. Therefore, a higher fatty acid concentration in the culture medium favors the MSC differentiation toward adipocytes, even in osteogenic medium.

On the other hand, the presence of AA (or such fatty acid plus some of the tested omega-3 fatty acids) significantly increased the expression of the ppary 2 and $l p l$ adipogenic genes in the cell cultures induced to differentiate into osteoblasts, in relation to the control ones, mainly at day 21 of treatment (Fig. 3). The supplementation of AA alone considerably increased the expression of the $l p l$ gene at day 7 of treatment, in relation to the control cultures and, to a lesser extent, in relation to the tissue cultures supplemented with AA plus DHA (or AA plus EPA). These two latter treatments increased the expression of the ppar 2 gene at day 21 of adipogenic induction, in relation to the cultures supplemented with AA only (Fig. 3). 
(a)
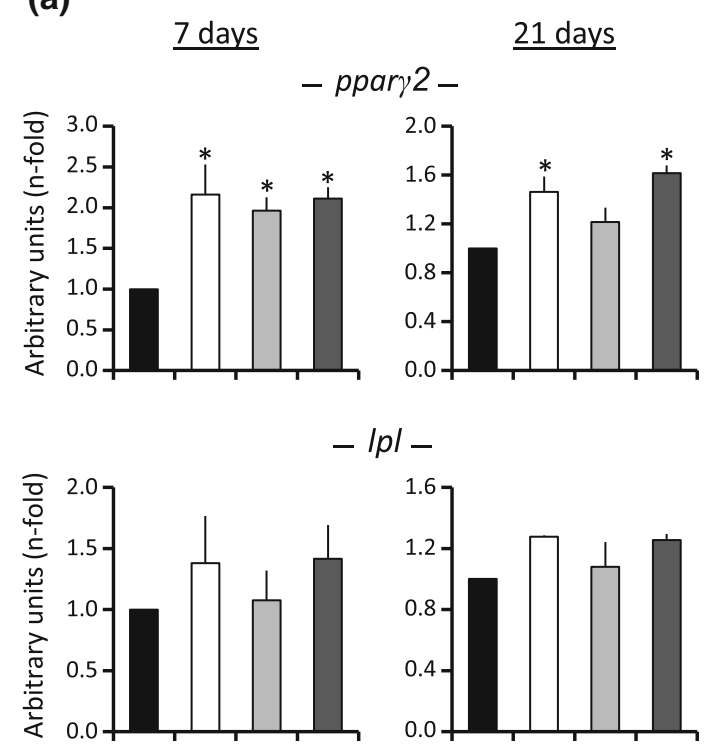

- gpd1
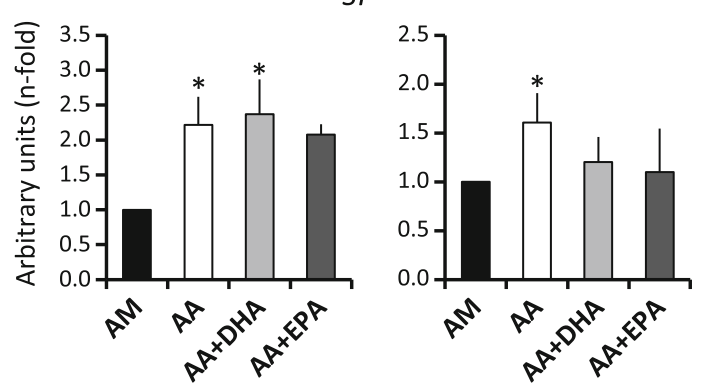

(b)
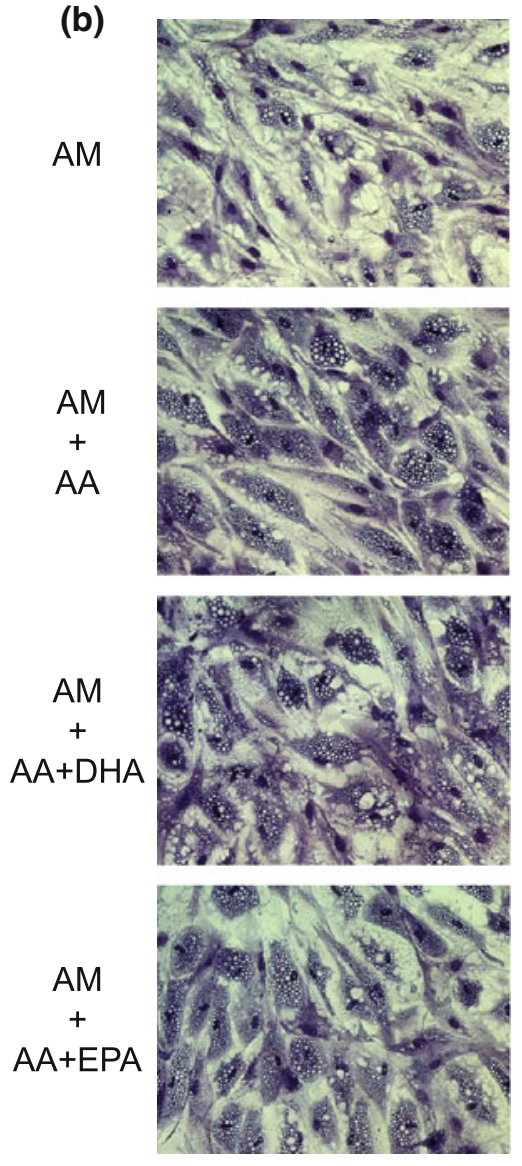

(c)

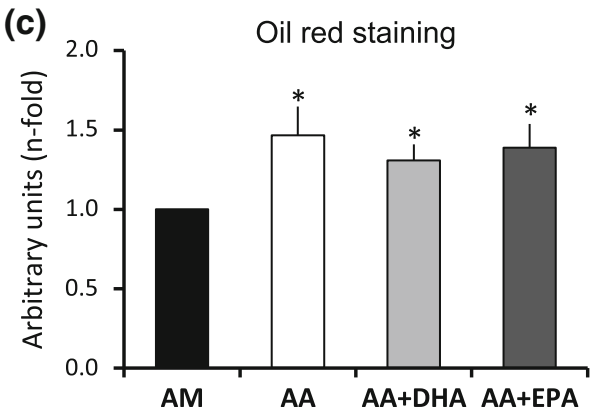

Fig. 1 Adipogenic differentiation. Adipogenic markers of the MSC induced to differentiate into adipocytes in adipogenic medium alone (AM), or supplemented with either $20 \mu \mathrm{M}$ of arachidonic acid (AA), $20 \mu \mathrm{M}$ of AA plus $20 \mu \mathrm{M}$ of DHA (AA + DHA) or $20 \mu \mathrm{M}$ of AA plus $20 \mu \mathrm{M}$ of EPA (AA + EPA). a Expression of the ppar 2 , lpl and gpdl adipogenic genes at 7 and 21 days after adipogenic differentiation. The values are relative to the AM control (value of 1).

Non-enzymatic hydroxyeicosatetraenoic acid concentration increases in culture media supplemented with arachidonic acid

Since the cell culture media were refreshed every $3-4$ days, the different cell culture media used in the experiments b Optical micrographs of crystal violet staining of the MSC induced to differentiate into adipocytes in the presence or absence of PUFA at day 21. c Quantification of the fat storage by the oil red staining and elution for the different treatments at day 21 of adipogenic induction. The values are relative to the AM control (value of 1 ). $* p<0.05$ in relation to $\mathrm{AM}$

without cells were incubated for 4 days, to check whether AA suffered some kind of alteration, including basal medium ( $\alpha$-MEM plus $10 \%$ FBS), adipogenic and osteogenic media, with or without $20 \mu \mathrm{M} \mathrm{AA}$, under the same conditions used for the cell cultures: $37{ }^{\circ} \mathrm{C}, 95 \%$ humidity and $5 \% \mathrm{CO}_{2}$. 
Fig. 2 Osteogenic differentiation. a Expression of runx 2, alp and $o c$ osteogenic genes in MSC induced to differentiate into osteoblasts in osteogenic medium alone (OM), or supplemented with either $20 \mu \mathrm{M}$ of arachidonic acid (AA), $20 \mu \mathrm{M}$ of AA plus $20 \mu \mathrm{M}$ of DHA (AA + DHA) or $20 \mu \mathrm{M}$ of AA plus $20 \mu \mathrm{M}$ of $\mathrm{EPA}(\mathrm{AA}+\mathrm{EPA})$, at days 7 and 21 of osteogenic differentiation. The values are relative to $\mathrm{OM}$ control (value of 1). b Optical micrographs of MSC induced to differentiate into osteoblasts at day 21 , showing the presence of adipocytes when culture medium was supplemented with $\mathrm{AA}, \mathrm{AA}+\mathrm{DHA}$ or

AA + EPA. c Quantification of adipocyte number for the different treatments at day 21 of osteogenic induction. $* p<0.05$ in relation to $\mathrm{OM} ; \# p<0.05$ in relation to $\mathrm{AA}$

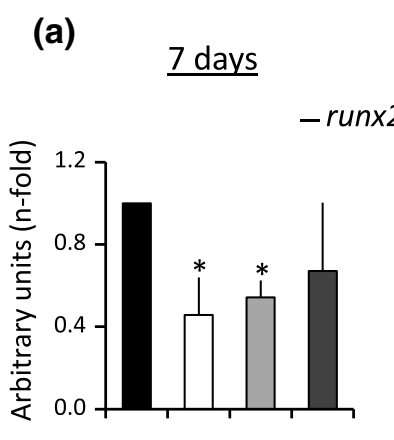

(b)

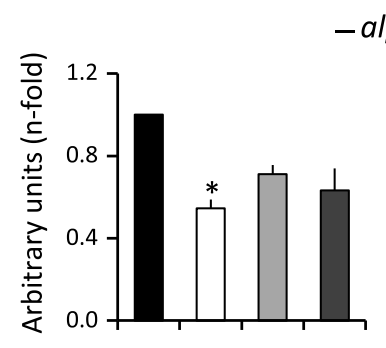

$\underline{21 \text { days }}$

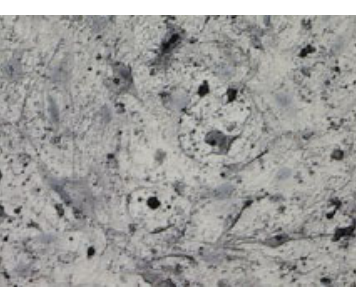

OM

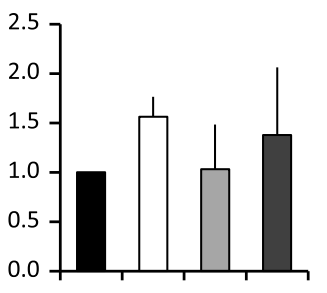

-alp -
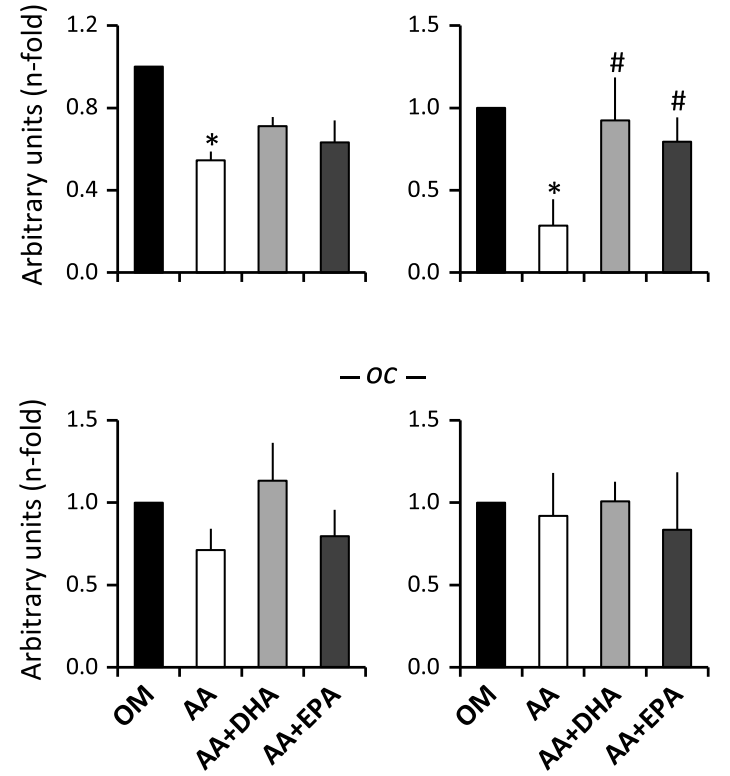

$O C-$
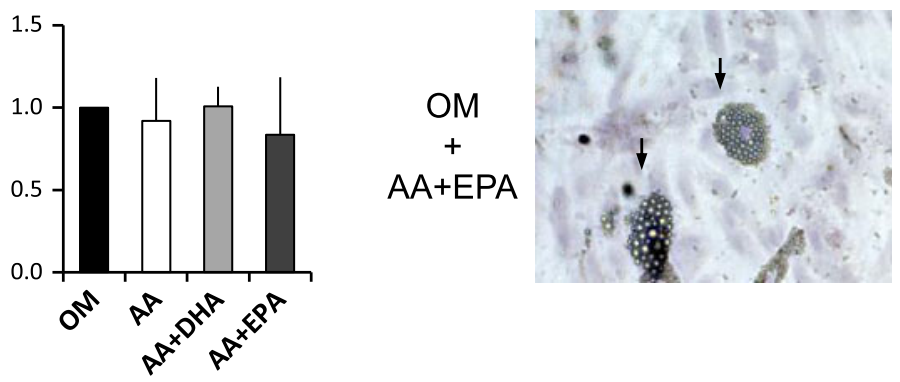

(c)

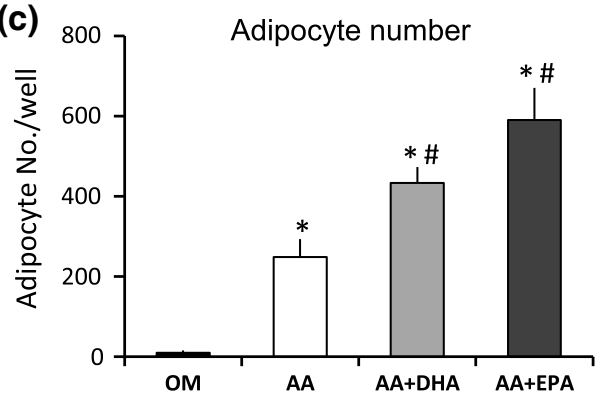

Interestingly, the concentration of the five HETE under study (5-, 8-, 11-, 12- and 15-HETE) increased more than 10 times when AA-containing media without cells were incubated under the same conditions used for the cell cultures (Fig. 4). This behavior should be due to a nonenzymatic peroxidation of the AA, since no cells were present. Additionally, the production of the studied HETE showed no significant concentration differences between the studied cell culture media supplemented with AA (Fig. 4).
Effect of the MSC differentiation into osteoblasts or adipocytes on the hydroxyeicosatetraenoic acid concentration on cell culture media supplemented with arachidonic acid

The MSC grown in different media (basal, adipogenic or osteogenic) were treated with AA, AA plus DHA, or AA plus EPA. The concentrations of 5-, 8-, 11-, 12- and 15-HETE were determined at days 10 and 21 after treatment. The concentration of HETE in the cell culture media 


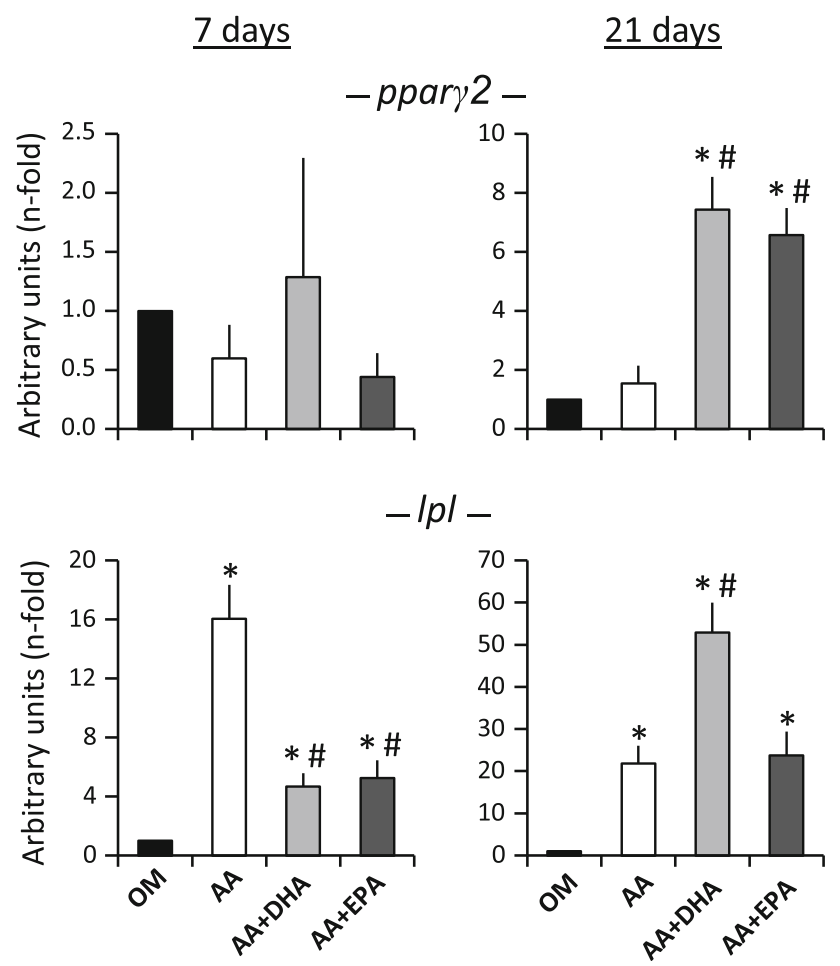

Fig. 3 Adipogenic gene expression. Quantification of expression of the ppary 2 and $l p l$ adipogenic genes in MSC induced to differentiate into osteoblasts in osteogenic medium alone $(\mathrm{OM})$, or supplemented with either $20 \mu \mathrm{M}$ of arachidonic acid (AA), $20 \mu \mathrm{M}$ of AA plus $20 \mu \mathrm{M}$ of DHA (AA + DHA) or $20 \mu \mathrm{M}$ of AA plus $20 \mu \mathrm{M}$ of EPA $(\mathrm{AA}+\mathrm{EPA})$ at days 7 and 21 of osteogenic differentiation. The values are relative to the $\mathrm{OM}$ control (value of 1 ). ${ }^{*} p<0.05$ in relation to $\mathrm{OM} ; \# p<0.05$ versus to $\mathrm{AA}$

was usually higher in the basal culture medium for all treatments, mainly after 21 days (Fig. 5). Thus, the AA present in such media increased the concentrations of the different HETE, in relation to the control untreated cultures: about 27 times for 12-HETE and more than 125 times for 5-HETE. The joint supplementation of AA with either DHA or EPA did not significantly alter the total HETE concentration, although a tendency to lower 5-, 8-, 11- and 12-HETE concentrations was observed in the cultures treated with AA and DHA, as compared to those supplemented with AA only (Fig. 5).

The HETE concentration in the media of the cell cultures induced to differentiate into adipocytes was significantly lower than in the average of the cell cultures maintained in basal or osteoblastic media, mainly at day 21 of treatment (Fig. 5). No significant differences in the concentration of 5- or 12-HETE were observed in the culture media of MSC induced to differentiate into adipocytes in the presence of AA, when compared to untreated controls. Besides, the 5-HETE was almost undetectable in the cultures maintained in adipogenic media for all treatments (Fig. 5). The concentration of 8-HETE in the media of the cell cultures induced to differentiate into adipocytes was similar to the one in basal or osteoblastic media at day 10. Yet, such concentration was significantly reduced in the adipogenic media at day 21 , in relation to both the average at day 10 and the other two kinds of culture media (Fig. 5). A concentration change was also observed for 11-, 12- and 15-HETE at day 21, showing a reduction in such products in the cell culture medium with maturation and differentiation (Fig. 5). When the adipogenic medium containing AA was also supplemented with DHA or EPA, the latter one reduced the 8-, 11- and 15-HETE concentrations in relation to the cultures supplemented with AA only (Fig. 5).

The presence of AA (alone or supplemented with either DHA or EPA) also generated an increase in the concentration of all the studied HETE in the media of cell cultures induced to differentiate into osteoblasts, in relation to the controls maintained in osteoblastic medium only. The concentrations of 5-, 8-, 11- and 12-HETE were similar to the ones for cultures in basal medium at day 10. Nevertheless, the concentrations of such products in the osteoblastic medium were significantly higher than those corresponding to the adipogenic medium, except in the case of 8-HETE (Fig. 5). The concentration of all studied HETE was reduced in all treatments at day 21, but it was always higher than the average for the MSC cultures induced to differentiate into adipocytes (Fig. 5). Therefore, the cultures induced to differentiate into osteoblast also reduced the concentration of the studied HETE in the culture medium, with the progression of the differentiation. The presence of AA plus EPA or DHA in the osteoblastic medium caused a reduction in 11-HETE concentration at day 10, but not at day 21. The presence of EPA also reduced the 8- and 12-HETE concentrations at both times, as compared to cultures treated with AA only (Fig. 5).

\section{Lipoxygenase 15B (alox 15b) gene expression}

Lipoxygenases catalyze the enzymatic production of HETE. The expression of alox 5, alox 12, alox 15 and alox $15 b$ genes was analyzed by QRT-PCR, to evaluate their putative implication in the observed variations of the different HETE concentrations in the different culture media and treatments, in the presence of AA plus DHA or EPA. Only the alox $15 b$ gene generated PCR amplifications, probably due to the low expression of the other ones. The lowest expression for such gene for all treatments at days 7 and 21 was observed in the cell cultures maintained with basal medium (Fig. 6). The expression of the cultures induced to differentiate into adipocytes was in general higher for all treatments at day 7 , in relation to other cultures. The presence of AA in the adipogenic medium generated the highest induction of such gene at day 21 . 

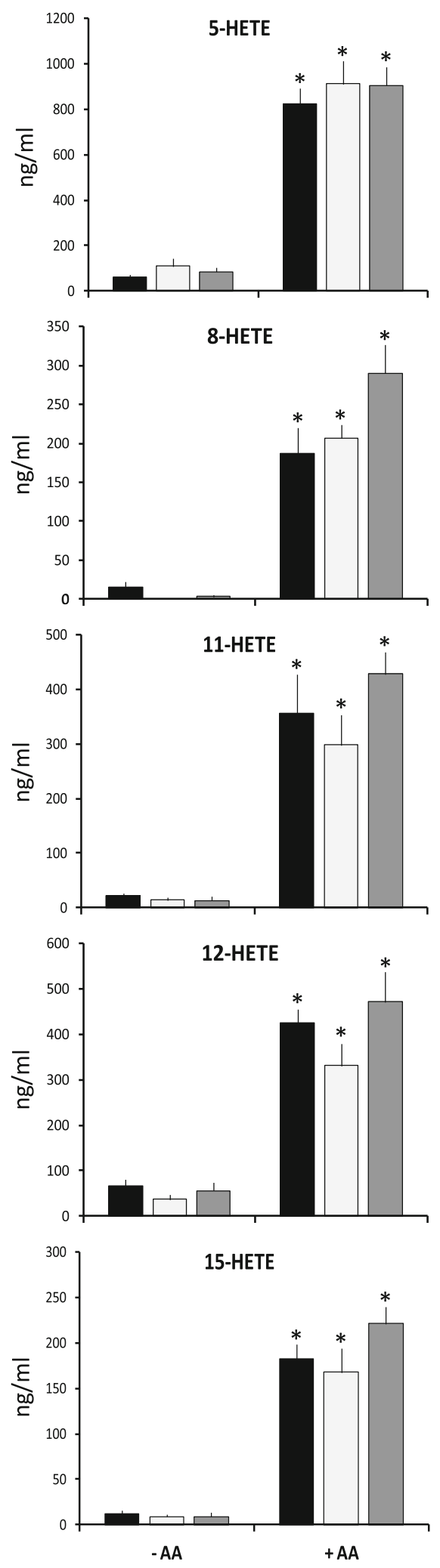

Basal culture medium Adipogenic culture medium Osteoblastic culture medium
Fig. 4 HETE concentrations in the culture medium in the absence of cells. Quantification of 5-, 8-, 11-, 12- and 15-HETE concentrations in the basal, adipogenic and osteogenic medium alone (-AA) or supplemented with $20 \mu \mathrm{M}$ of arachidonic acid (+AA), in the absence of cells, at day 4 of incubation in the same cell culture conditions. $p<0.05$ in relation to the same medium without the AA

On the other hand, the cells induced to differentiate into osteoblasts in the presence of AA plus EPA showed a significant induction of alox $15 \mathrm{~b}$ gene expression at day 21 of treatment, in relation to cultures maintained in adipogenic or basal media (Fig. 6). This figure highlights that the expression of such gene was always higher at day 21 than at day 7 for all treatments, mainly in cultures induced to differentiate into adipocytes or osteoblasts. These results, together with the fact that cultures maintained in the basal medium exhibited a lower gene expression, demonstrate a negative correlation between the different HETE concentrations in the culture media and the alox $15 \mathrm{~b}$ gene expression. This suggests that the different HETE quantified in the different culture media derived, at least in part, from other AA oxidation source.

\section{Discussion}

Omega- 6 and omega-3 fatty acids, as well as the eicosanoids derived from their metabolism, may work as regulatory molecules of different metabolic and signaling networks in the cell. The biological activity of such fatty acids has been related to different biological processes, including cellular differentiation, inflammation, vascularization and tumorigenesis, among others (Kim et al. 2010; Qadhi et al. 2013; Shaikh et al. 2012). Therefore, they may be involved in different physiological aspects of the organism, suggesting that their dietary consumption should be equilibrated to avoid metabolic and physiological deregulations that eventually could cause different pathologies.

The modern Western dietary patterns are abundant in omega- 6 , yet deficient in omega- 3 fatty acids, resulting in a omega-6/omega-3 ratio $>10: 1$. Interestingly, it has been proposed that such high ratios might represent a significant risk factor in some life-threatening diseases (Simopoulos 2008), including the loss of bone mass density (Jarvinen et al. 2012). Even more, recent animal studies have shown that diets with both omega- 6 and omega- 3 fatty acids may alter the tissue lipidic composition, with a potentially healthier production of inflammatory cytokines when such ratio approaches the value of one (Blanchard et al. 2013; Duan et al. 2013). Nevertheless, the significance of these findings in the development of some diseases remains to be 
Fig. 5 HETE concentrations in the culture medium in the presence of cells. Quantification of 5-, 8-, 11-, 12- and 15-HETE concentrations in basal, adipogenic and osteogenic medium, corresponding to cell cultures maintained in the absence of PUFA (controls) or treated with $20 \mu \mathrm{M}$ of arachidonic acid (AA), $20 \mu \mathrm{M}$ of AA plus $20 \mu \mathrm{M}$ of DHA (AA + DHA) or $20 \mu \mathrm{M}$ of AA plus $20 \mu \mathrm{M}$ EPA (AA + EPA), at days 10 and 21 after differentiation start. $* p<0.05$ in relation to the cultures maintained in basal medium for the same treatments and time; $\# p<0.05$ in relation to the cultures maintained in same medium and treatments at day 10
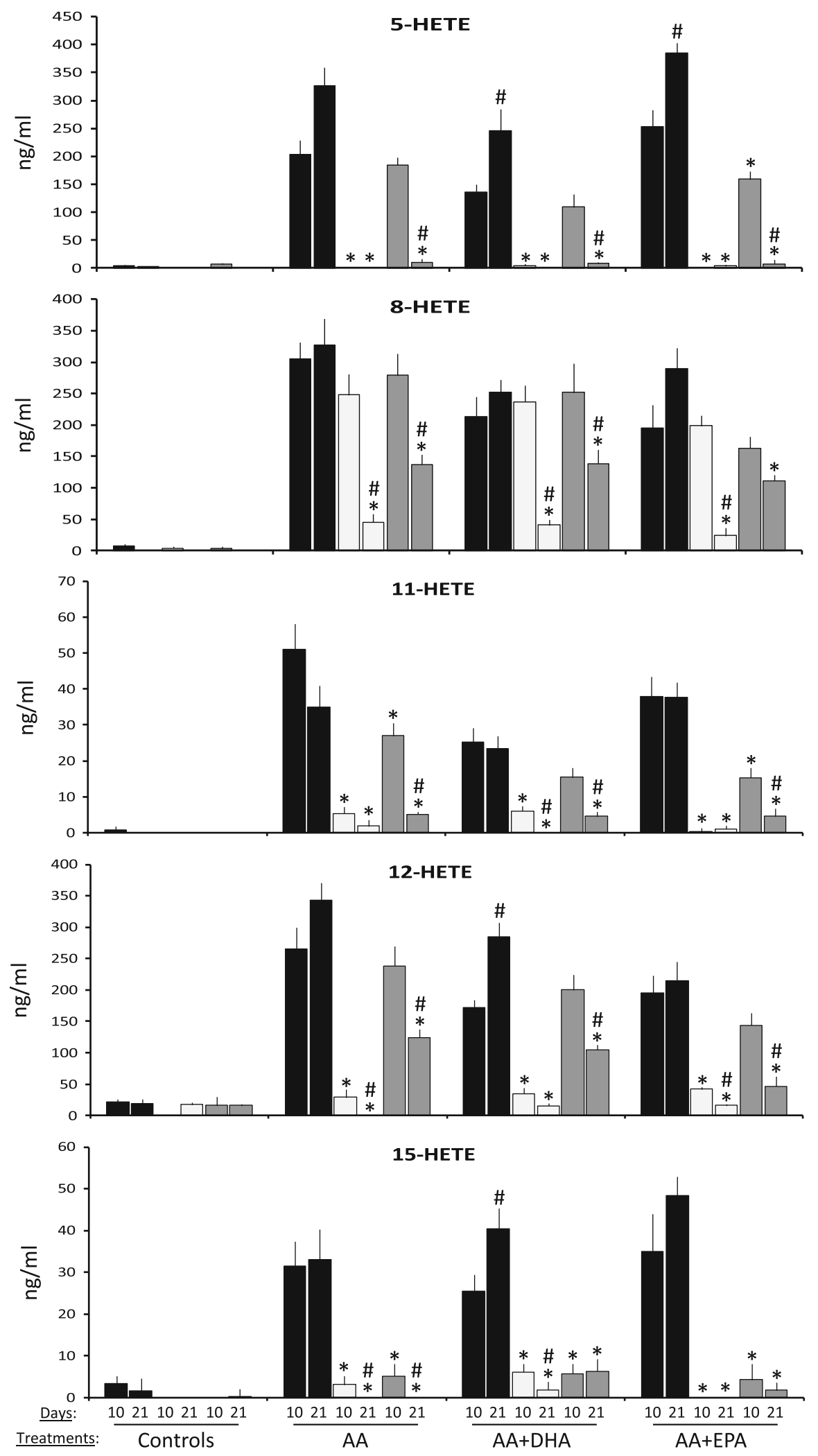

Cells not induced to differentiate

Cells induced to differentiate into adipocytes

Cells induced to differentiate into osteoblast 


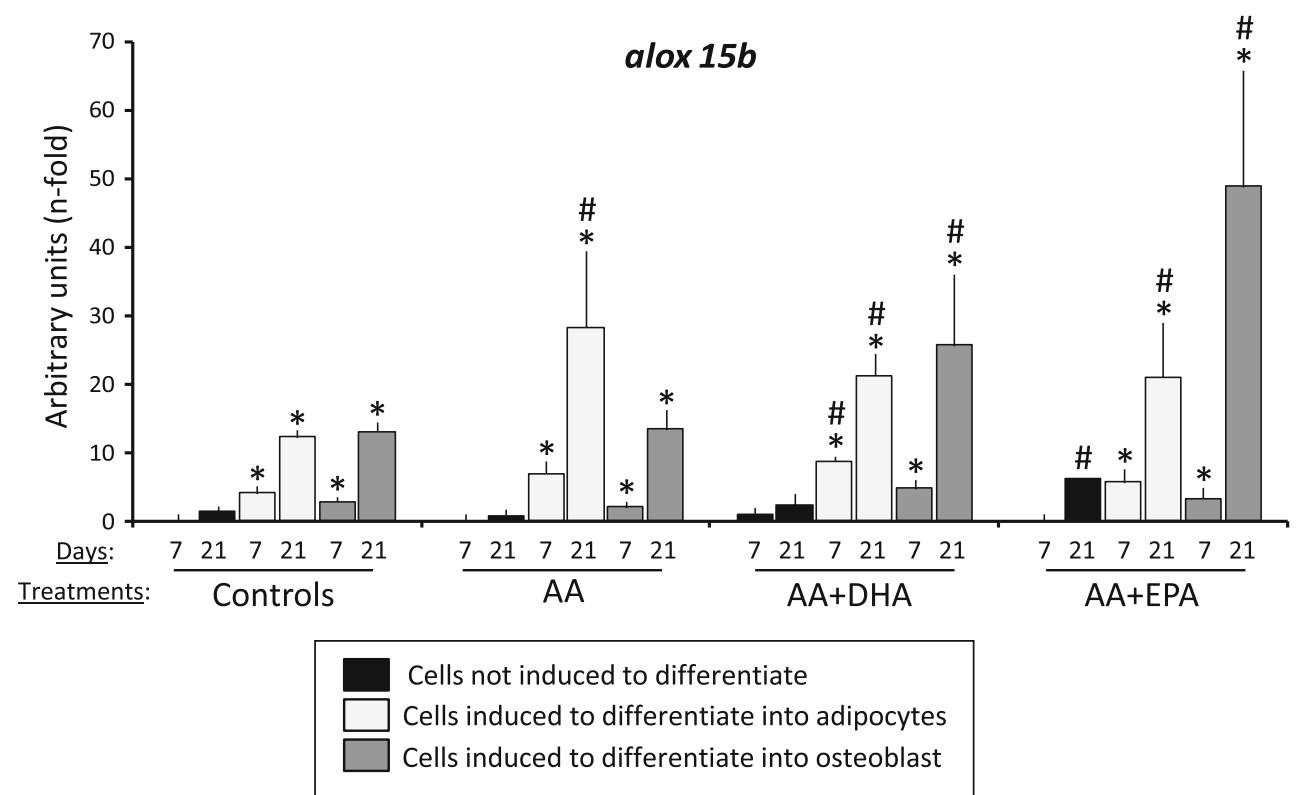

Fig. 6 Lipoxygenase gene expression. Quantification of expression of the alox $15 \mathrm{~b}$ gene in MSC cultures maintained in basal, adipogenic or osteogenic medium, without treatment (controls) or treated with $20 \mu \mathrm{M}$ of arachidonic acid (AA), $20 \mu \mathrm{M}$ of AA plus $20 \mu \mathrm{M}$ of DHA

further studied and validated in well-qualified in vitro/ in vivo experimental models, as well as in epidemiological and intervention studies.

We have previously reported that the AA favors the MSC differentiation into adipocytes (Casado-Diaz et al. 2013), demonstrating that both 20 and $40 \mu \mathrm{M}$ of AA enhance the adipogenesis, with a $40 \mu \mathrm{M}$ concentration even inhibiting the osteoblastogenesis. We have further studied the $20 \mu \mathrm{M}$ concentration in the present work, since when the AA is added with DHA or EPA to the culture medium, the PUFA concentration in the medium reaches $40 \mu \mathrm{M}$. Yet, such a $40 \mu \mathrm{M}$ AA concentration with any of such PUFA would have generated a final culture medium concentration of $80 \mu \mathrm{M}$, which is supraphysiological and cytotoxic for the MSC in our tests (data not shown). This is in agreement with results obtained for other cellular types in which PUFA concentrations higher than $60 \mu \mathrm{M}$ in the culture medium reduced both the cellular viability and proliferation rate (Di Nunzio et al. 2011; Shirota et al. 2005). The present work demonstrates that AA adipogenesis enhancement is not reduced by the joint treatment of AA plus DHA or AA plus EPA at the concentrations used in MSC induced to differentiate into adipocytes. Likewise, although the joint presence of AA plus DHA or EPA increased the expression of alp gene expression (osteoblastogenesis marker) in cultures induced to differentiate into osteoblasts, they also increased the expression of the ppary gene (adipogenic marker) and thus the appearance of adipocytes in such cultures as a result. Therefore, the addition
(AA + DHA) or $20 \mu \mathrm{M}$ of AA plus $20 \mu \mathrm{M}$ of EPA (AA + EPA), at days 7 and 21 of differentiation. $* p<0.05$ in relation to cultures maintained in basal medium for the same treatments and time; $\# p<0.05$ in relation to respective culture control

of $20 \mu \mathrm{M}$ of AA plus the same concentration of DHA or EPA generates a $40 \mu \mathrm{M}$ PUFA concentration in the culture medium, not reducing the effect of the AA on adipogenesis in our experimental model, and even enhancing it in the MSC cultures induced to differentiate into osteoblasts.

These results suggest that even with an 1:1 omega-6/ omega-3 ratio as in this case, the increase in the PUFA concentration in the culture media (when DHA or EPA are added) favors the adipocyte generation. In fact, the presence of fatty acids in the culture medium is essential for the adipogenic differentiation. Thus, 3T3-L1 pre-adipocytes do not differentiate into adipocytes in the presence of delipidated (delipid) medium unless PPAR $\gamma$ agonists are added (Chawla and Lazar 1994). Besides, when the cultures of this cell line (induced to differentiate into adipocytes with dexamethasone and insulin) are supplemented with omega-6 or omega-3 fatty acids, the differentiation rate increases (Madsen et al. 2005). Additionally, we have demonstrated that there is an increase in the number of adipocytes when the MSC cultures (induced to differentiate into osteoblasts) are supplemented with $40 \mu \mathrm{M}$ of DHA or EPA (CasadoDiaz et al. 2013). On the other hand, it is known that the consumption of low concentrations of PUFA is positive for bone formation, albeit a high consumption may not have beneficial effects (Mollard et al. 2005).

We have found that AA is oxidized in the culture media (without cells) by non-enzymatic pathways, as demonstrated by the increase in hydroxyeicosatetraenoic acids under such experimental conditions. In fact, the non-enzymatic 
peroxidation is a known process (Niki et al. 2005), mainly involving unsaturated fats (Spiteller 2010), being further enhanced in our experiments, due to the culture medium incubation in the presence of atmospheric oxygen $(21 \%)$. The concentration of the five studied HETE did not significantly change in the three culture media used (basal, adipogenic and osteogenic) after incubation in the presence of AA without cells. As expected, the HETE concentration underwent important changes in the presence of cells, depending on the medium capability to induce (or not) the MSC differentiation, with significant differences in most cases, in relation to the differentiation stage (time). These results show that the MSC metabolize AA and derived products, depending on their physiological state (differentiating or not) and on their differentiation stage.

PUFA play an important role in the MSC differentiation into adipocytes (Madsen et al. 2005). A fat-rich diet increases the expression of the genes encoding the C/EBP $\alpha$ and PPAR $\gamma 2$ transcription factors, which are inducers of adipogenic differentiation. In this sense, the products derived from the PUFA metabolism are considered direct inducers of the ppary gene expression (Madsen et al. 2005). Therefore, they may produce different effects, depending on the nature of the involved PUFA. Thus, the fat storage is reduced in rodents if the diet is richer in n-3 PUFA, when compared to a richer one in n-6 PUFA (Takahashi and Ide 2000).

PUFA metabolism produces eicosanoids by means of lipoxygenases, cyclooxygenases and cytochrome P450 (CYP) epoxygenases. All such enzymes use AA as substrate. The importance of such PUFA in MSC induced to differentiate into adipocytes is revealed by the fact that in the differentiation process, the specific AdPLA phospholipase is synthesized, the inhibition of which increases lipolysis and decreases obesity (Jaworski et al. 2009). The phospholipase generates AA, which is further metabolized into products that may modulate the MSC differentiation pathways. Besides, the eicosanoids produced by the 20-carbon PUFA oxidation can be esterified in the cellular membrane with phospholipids, where they can be stored and also carry out different biological activities (Hammond and O'Donnell 2012). The HETE studied in this work are among such products, which can be generated by both nonenzymatic oxidation, as well as by the enzymatic activity of the lipoxygenases (5-, 8-, 12- and 15-HETE), cyclooxygenases (11- and 15-HETE) and CYP proteins (11-HETE) (Moreno 2009). On the other hand, HETE may modulate the cellular cycle, inhibiting the apoptosis and being either mitogenic (5-, 12- and 15-HETE) or anti-mitogenic (8-, 11-, 12- and 15-HETE) agents in different cellular types (Moreno 2009). This suggests that they alone (or the products derived from their metabolism) may be involved in different cellular processes, such as differentiation.
Our results show that 8-HETE was the only studied HETE present at high concentrations at day 7 after MSC induction to differentiation, in adipogenic medium supplemented with PUFA. This suggests that such HETE may be a mediator of the adipogenesis activation in our experimental model. This is in agreement with the fact that 8-HETE is a PPAR $\alpha$ activator, and it can induce the 3T3L1 pre-adipocyte differentiation toward adipocytes ( $\mathrm{Yu}$ et al. 1995). The studied HETE in cells induced to differentiate into osteoblasts were present at higher concentrations than in adipogenic media, in general. Some HETE may modulate osteoblastogenesis. Thus, 12- and 15-HETE may inhibit differentiation and promote apoptosis of osteoblasts (Almeida et al. 2009). Additionally, the 5-HETE may inhibit osteoblastogenesis (Traianedes et al. 1998).

Lipoxygenases represent the main enzymatic activity for HETE production and further metabolism. The relevance of such enzymes for MSC differentiation into adipocytes has been demonstrated in 3T3-L1 pre-adipocytic cells from mouse. Thus, adipogenesis is inhibited in such cells when they are treated with the nordihydroguaiaretic acid (NDGA) and baicalein (BC) lipoxygenase inhibitors during the first four days of differentiation induction (Madsen et al. 2003). More recently, it has been demonstrated that hepoxilins, which are produced by the eLOX2 lipoxygenase activity, activate PPAR $\gamma$ and adipogenesis in the 3T3L1 cells (Hallenborg et al. 2010). Additionally, other compounds produced by lipoxygenases, such as the leukotriene B4 (LTB4), which is generated by the 5-LOX enzyme, are an adipogenesis inhibitor via binding to its receptor (BLT) (Hirata et al. 2012).

We have not found any lox-5 gene expression, and 5-HETE was found at very low concentrations in the media of MSC cultures induced to differentiate into adipocytes. We have detected alox $15 b$ lipoxygenase gene expression, which increases in cell cultures supplemented with PUFA and induced to differentiate into adipocytes, mainly at day 21 , which is when drastic reductions in the concentrations of the five studied HETE in culture medium were also found. This is positively correlated with an increase in expression of adipogenic genes and the formation of fat vesicles in the cells of such cultures. Therefore, the increase in adipogenesis can be correlated with an increase in metabolism of added PUFA or their metabolites inside the cells, producing a reduction in free metabolites in culture medium, which must be mediated, at least partially, by the ALOX 15B enzymatic activity.

We have also found that the addition of PUFA to the culture medium of the MSC induced to differentiate into osteoblasts increases the number of adipocytes and ppar 2 gene expression. This is in agreement with the effect of lipid oxidation on bone metabolism, in which lipoxygenase activity is partially responsible for the loss of bone mass 
with aging. Thus, $B$-catenin does not bind $\mathrm{T}$ cell-specific transcription factors to induce the MSC differentiation into osteoblasts, due to the increase in oxidative stress caused by such enzyme, but instead it binds to the forkhead box $\mathrm{O}$ (FoxO) transcription factors. This induces ppar 22 gene and therefore triggers the MSC differentiation into adipocytes (Almeida et al. 2009).

Our data show that the 15-HETE concentration is low in culture media supplemented with PUFA and induced to differentiate into osteoblasts, in comparison with the control cultures not induced to differentiate. Besides, it diminishes at day 21, as the other studied HETE. We have found that the expression of the alox $15 b$ gene increased at such time, mainly in cultures supplemented with AA plus DHA, as well as AA plus EPA. This is in agreement with the fact that a higher number of adipocytes were found in such two treatments. Thus, our results show a correlation between the induction of the alox $15 \mathrm{~b}$ gene expression and the adipogenesis. This is in agreement with the results obtained by other authors in mouse, showing that an increase in the alox 15 gene expression in osteoblasts is negatively correlated with bone mass (Klein et al. 2004).

As expected, MSC induced to differentiate into osteoblasts or adipocytes show a different AA metabolism than the control uninduced ones. Thus, our results show that the presence of AA in culture medium generates an accumulation of the studied HETE in the latter, in contrast to differentiating MSC cultures. Yet, such non-differentiated cultures have a low alox $15 b$ gene expression. Therefore, our results suggest that such products should derive from a non-enzymatic oxidation of AA, rather than from lipoxygenase activity. Thus, the uninduced MSC should have a lower rate of enzymatic metabolism of such products via lipoxygenases, in comparison with the ones induced to differentiate. This is in agreement with the fact that (in contrast to the ones induced to differentiate into adipocytes), uninduced MSC exhibit a higher CYP450 monooxygenase expression, which is responsible for the biosynthesis of the epoxyeicosatrienoic acids (EET) from AA, being the former inhibitors of the adipogenesis (Kim et al. 2010).

In conclusion, our results indicate that there are differences in PUFA metabolism between uninduced MSC and those induced to differentiation into osteoblasts or adipocytes. Addition of PUFA to the former generates an increase in their metabolites in the culture medium, indicating that they are not further metabolized (or that such metabolism is rather slow). This is in agreement with the low lipoxygenase alox $15 \mathrm{~b}$ gene expression, in relation to cultures induced to differentiate. Interestingly, this may represent an MSC physiological strategy for maintaining their undifferentiated state in the absence of other more specific differentiation-inducing agents. On the other hand, the PUFA metabolism is triggered in the MSC induced to differentiate into osteoblasts or adipocytes; and mostly in the latter, as revealed by the increase in the alox $15 \mathrm{~b}$ gene expression and the drastic reduction in PUFA metabolites in culture media. In fact, the increase in such metabolism (found in MSC induced to differentiate into osteoblasts or adipocytes) is correlated with an increase in adipogenesis.

Therefore, a high intake of PUFA (omega- 6 or other activators of the lipoxygenase activity) may have a negative impact on the bone formation. This suggests that nutritional alternatives are needed for improving nonoptimal omega-6/omega-3 ratios and that an increase in the omega-3 of fish (EPA and DHA) or plant (linolenic acid) origin in the diet may be regarded as a valuable strategy for the prevention of and treatment for bone metabolism pathologies, such as osteoporosis (Hogstrom et al. 2007; Weiss et al. 2005). However, taking the above into account, this might not be so simple. Probably, specific dietary pharmacological recommendations should be additionally established, as well as considerations of possible genetic factors that may modulate the requirements of these nutrients, in order to minimize the induction of adipocyte formation and optimize the formation of osteoblasts. In any case, further studies are needed to shed new light on the more controversial aspects that have arisen in relation to this subject and to validate the proposed hypotheses.

Acknowledgments This work was supported by the "Grupo CTS413" of "Junta de Andalucía", RETICEF and the project PI081692 of the "Ministerio de Ciencia e Innovación" (MICINN) of Spain.

\section{Conflict of interest None.}

Ethical standards This article does not contain any studies with human or animal subjects performed by any of the authors.

\section{References}

Almeida M, Ambrogini E, Han L, Manolagas SC, Jilka RL (2009) Increased lipid oxidation causes oxidative stress, increased peroxisome proliferator-activated receptor-gamma expression, and diminished pro-osteogenic Wnt signaling in the skeleton. J Biol Chem 284:27438-27448

Ban K, Sprunt JM, Martin S, Yang P, Kozar RA (2011) Glutamine activates peroxisome proliferator-activated receptor-gamma in intestinal epithelial cells via 15-S-HETE and 13-OXO-ODE: a novel mechanism. Am J Physiol Gastrointest Liver Physiol 301:G547-G554

Blanchard H, Pedrono F, Boulier-Monthean N, Catheline D, Rioux V, Legrand P (2013) Comparative effects of well-balanced diets enriched in alpha-linolenic or linoleic acids on LC-PUFA metabolism in rat tissues. Prostaglandins Leukot Essent Fatty Acids 88:383-389

Casado-Diaz A, Santiago-Mora R, Dorado G, Quesada-Gomez JM (2013) The omega-6 arachidonic fatty acid, but not the omega-3 fatty acids, inhibits osteoblastogenesis and induces adipogenesis 
of human mesenchymal stem cells: potential implication in osteoporosis. Osteoporos Int 24:1647-1661

Chakrabarti SK, Cole BK, Wen Y, Keller SR, Nadler JL (2009) 12/15-lipoxygenase products induce inflammation and impair insulin signaling in 3T3-L1 adipocytes. Obesity (Silver Spring) 17:1657-1663

Chawla A, Lazar MA (1994) Peroxisome proliferator and retinoid signaling pathways co-regulate preadipocyte phenotype and survival. Proc Natl Acad Sci USA 91:1786-1790

Chen TY, Zhang ZM, Zheng XC, Wang L, Huang MJ, Qin S, Chen J, Lai PL, Yang CL, Liu J, Dai YF, Jin DD, Bai XC (2013) Endogenous n-3 polyunsaturated fatty acids (PUFAs) mitigate ovariectomyinduced bone loss by attenuating bone marrow adipogenesis in FAT1 transgenic mice. Drug Des Dev Ther 7:545-552

Comba A, Pasqualini ME (2009) Primers on molecular pathwayslipoxygenases: their role as an oncogenic pathway in pancreatic cancer. Pancreatology 9:724-728

Di Mari JF, Saada JI, Mifflin RC, Valentich JD, Powell DW (2007) HETEs enhance IL-1-mediated COX-2 expression via augmentation of message stability in human colonic myofibroblasts. Am J Physiol Gastrointest Liver Physiol 293:G719-G728

Di Nunzio M, Valli V, Bordoni A (2011) Pro- and anti-oxidant effects of polyunsaturated fatty acid supplementation in HepG2 cells. Prostaglandins Leukot Essent Fatty Acids 85:121-127

Duan Y, Li F, Li L, Fan J, Sun X, Yin Y (2013) n-6:n-3 PUFA ratio is involved in regulating lipid metabolism and inflammation in pigs. Br J Nutr 1-7

Ferreiro-Vera C, Mata-Granados JM, Priego-Capote F, QuesadaGomez JM, Luque de Castro MD (2011) Automated targeting analysis of eicosanoid inflammation biomarkers in human serum and in the exometabolome of stem cells by SPE-LC-MS/MS. Anal Bioanal Chem 399:1093-1103

Hallenborg P, Jorgensen C, Petersen RK, Feddersen S, Araujo P, Markt P, Langer T, Furstenberger G, Krieg P, Koppen A, Kalkhoven E, Madsen L, Kristiansen K (2010) Epidermis-type lipoxygenase 3 regulates adipocyte differentiation and peroxisome proliferator-activated receptor gamma activity. Mol Cell Biol 30:4077-4091

Hammond VJ, O’Donnell VB (2012) Esterified eicosanoids: generation, characterization and function. Biochim Biophys Acta 1818:2403-2412

Hirata K, Katayama K, Nakajima A, Takada K, Kamisaki Y, Wada K (2012) Role of leukotriene B(4) receptor signaling in human preadipocyte differentiation. Biochem Biophys Res Commun 429:197-203

Hogstrom M, Nordstrom P, Nordstrom A (2007) n-3 Fatty acids are positively associated with peak bone mineral density and bone accrual in healthy men: the NO2 Study. Am J Clin Nutr 85:803-807

Jarvinen R, Tuppurainen M, Erkkila AT, Penttinen P, Karkkainen M, Salovaara K, Jurvelin JS, Kroger H (2012) Associations of dietary polyunsaturated fatty acids with bone mineral density in elderly women. Eur J Clin Nutr 66:496-503

Jaworski K, Ahmadian M, Duncan RE, Sarkadi-Nagy E, Varady KA, Hellerstein MK, Lee HY, Samuel VT, Shulman GI, Kim KH, de Val S, Kang C, Sul HS (2009) AdPLA ablation increases lipolysis and prevents obesity induced by high-fat feeding or leptin deficiency. Nat Med 15:159-168

Kim DH, Vanella L, Inoue K, Burgess A, Gotlinger K, Manthati VL, Koduru SR, Zeldin DC, Falck JR, Schwartzman ML, Abraham NG (2010) Epoxyeicosatrienoic acid agonist regulates human mesenchymal stem cell-derived adipocytes through activation of HO-1-pAKT signaling and a decrease in PPARgamma. Stem Cells Dev 19:1863-1873

Klein RF, Allard J, Avnur Z, Nikolcheva T, Rotstein D, Carlos AS, Shea M, Waters RV, Belknap JK, Peltz G, Orwoll ES (2004)
Regulation of bone mass in mice by the lipoxygenase gene Alox 15. Science 303:229-232

Lopez IP, Marti A, Milagro FI, Zulet Md Mde L, Moreno-Aliaga MJ, Martinez JA, De Miguel C (2003) DNA microarray analysis of genes differentially expressed in diet-induced (cafeteria) obese rats. Obes Res 11:188-194

Madsen L, Petersen RK, Sorensen MB, Jorgensen C, Hallenborg P, Pridal L, Fleckner J, Amri EZ, Krieg P, Furstenberger G, Berge RK, Kristiansen K (2003) Adipocyte differentiation of 3T3-L1 preadipocytes is dependent on lipoxygenase activity during the initial stages of the differentiation process. Biochem J 375:539-549

Madsen L, Petersen RK, Kristiansen K (2005) Regulation of adipocyte differentiation and function by polyunsaturated fatty acids. Biochim Biophys Acta 1740:266-286

Moerman EJ, Teng K, Lipschitz DA, Lecka-Czernik B (2004) Aging activates adipogenic and suppresses osteogenic programs in mesenchymal marrow stroma/stem cells: the role of PPARgamma2 transcription factor and TGF-beta/BMP signaling pathways. Aging Cell 3:379-389

Mollard RC, Kovacs HR, Fitzpatrick-Wong SC, Weiler HA (2005) Low levels of dietary arachidonic and docosahexaenoic acids improve bone mass in neonatal piglets, but higher levels provide no benefit. J Nutr 135:505-512

Moon HJ, Kim TH, Byun DW, Park Y (2012) Positive correlation between erythrocyte levels of n-3 polyunsaturated fatty acids and bone mass in postmenopausal Korean women with osteoporosis. Ann Nutr Metab 60:146-153

Moreno JJ (2009) New aspects of the role of hydroxyeicosatetraenoic acids in cell growth and cancer development. Biochem Pharmacol 77:1-10

Needleman P, Turk J, Jakschik BA, Morrison AR, Lefkowith JB (1986) Arachidonic acid metabolism. Annu Rev Biochem 55:69-102

Niki E, Yoshida Y, Saito Y, Noguchi N (2005) Lipid peroxidation: mechanisms, inhibition, and biological effects. Biochem Biophys Res Commun 338:668-676

Pfaffl MW, Tichopad A, Prgomet C, Neuvians TP (2004) Determination of stable housekeeping genes, differentially regulated target genes and sample integrity: BestKeeper-excel-based tool using pair-wise correlations. Biotechnol Lett 26:509-515

Qadhi R, Alsaleh N, Samokhvalov V, El-Sikhry H, Bellenger J, Seubert JM (2013) Differential responses to docosahexaenoic acid in primary and immortalized cardiac cells. Toxicol Lett 219:288-297

Rahman MM, Bhattacharya A, Banu J, Kang JX, Fernandes G (2009) Endogenous n-3 fatty acids protect ovariectomy induced bone loss by attenuating osteoclastogenesis. J Cell Mol Med 13:1833-1844

Salari P, Rezaie A, Larijani B, Abdollahi M (2008) A systematic review of the impact of $n-3$ fatty acids in bone health and osteoporosis. Med Sci Monit 14:RA37-RA44

Schmitz G, Ecker J (2008) The opposing effects of n-3 and n-6 fatty acids. Prog Lipid Res 47:147-155

Shaikh SR, Jolly CA, Chapkin RS (2012) n-3 Polyunsaturated fatty acids exert immunomodulatory effects on lymphocytes by targeting plasma membrane molecular organization. Mol Aspects Med 33:46-54

Shillabeer G, Lau DC (1994) Regulation of new fat cell formation in rats: the role of dietary fats. J Lipid Res 35:592-600

Shirota T, Haji S, Yamasaki M, Iwasaki T, Hidaka T, Takeyama Y, Shiozaki H, Ohyanagi H (2005) Apoptosis in human pancreatic cancer cells induced by eicosapentaenoic acid. Nutrition 21:1010-1017

Simopoulos AP (2008) The importance of the omega-6/omega-3 fatty acid ratio in cardiovascular disease and other chronic diseases. Exp Biol Med (Maywood) 233:674-688 
Spiteller G (2010) Is lipid peroxidation of polyunsaturated acids the only source of free radicals that induce aging and age-related diseases? Rejuvenation Res 13:91-103

Takahashi Y, Ide T (2000) Dietary n-3 fatty acids affect mRNA level of brown adipose tissue uncoupling protein 1 , and white adipose tissue leptin and glucose transporter 4 in the rat. Br J Nutr 84:175-184

Traianedes K, Dallas MR, Garrett IR, Mundy GR, Bonewald LF (1998) 5-Lipoxygenase metabolites inhibit bone formation in vitro. Endocrinology 139:3178-3184
Wahli W, Michalik L (2012) PPARs at the crossroads of lipid signaling and inflammation. Trends Endocrinol Metab 23:351-363

Weiss LA, Barrett-Connor E, von Muhlen D (2005) Ratio of n-6 to n-3 fatty acids and bone mineral density in older adults: the Rancho Bernardo Study. Am J Clin Nutr 81:934-938

Yu K, Bayona W, Kallen CB, Harding HP, Ravera CP, McMahon G, Brown M, Lazar MA (1995) Differential activation of peroxisome proliferator-activated receptors by eicosanoids. J Biol Chem 270:23975-23983 